\title{
The MSC-MCF-7 Duet Playing Tumor Vasculogenesis and Angiogenesis onto the Chick Embryo Chorioallantoic Membrane
}

\author{
ŞERBAN COMŞA ${ }^{1,2}$, AMALIA-RALUCA CEAUȘU ${ }^{1,2}$, ROXANA POPESCU $^{3}$, \\ SIMONA SÂRB ${ }^{1}$, ANCA-MARIA CIMPEAN $^{1,2}$ and MARIUS RAICA ${ }^{1,2}$ \\ ${ }^{1}$ Department of Microscopic Morphology/Histology, \\ ${ }^{2}$ Angiogenesis Research Center, and \\ ${ }^{3}$ Department of Microscopic Morphology/Cell and Molecular Biology, \\ "Victor Babeş" University of Medicine and Pharmacy, Timişoara, Romania
}

\begin{abstract}
Background/Aim: Human mesenchymal stem cells (hMSC) represent a versatile cell population, able to modulate the tumor microenvironment. Our aim was to recreate an open scene for the in vivo interaction between hMSC and the MCF-7 breast cancer cells (MCF-7), in order to enlighten the intimate involvement of hMSC in tumor vasculogenesis and angiogenesis. Materials and Methods: hMSC and MCF-7 were seeded onto the chick embryo chorioallantoic membrane (CAM) and incubated for 7 days. Consecutively, the morphology and the immunohistochemical profile of CAM were assessed. Results: Following this complex interaction, MCF-7 acquired a more aggressive phenotype, hMSC switched to a vascular precursor phenotype, while CAM underwent a major reset to an earlier stage, with hotspots of angiogenesis, vasculogenesis and hematopoiesis. Conclusion: The hallmark of this study was the establishment of a veritable in vivo experimental model of MSC involvement in tumor vasculogenesis and angiogenesis, allowing further analysis in the field.
\end{abstract}

As tumor vasculature has been thought to emerge by expansion of the host vasculature through postnatal sprouting of endothelial cells (angiogenesis), it was proposed that inhibiting angiogenesis would stop tumor growth, but it turned out that tumor vessels form through vasculogenesis,

This article is freely accessible online.

Correspondence to: Anca Maria Cîmpean, Department of Histology, "Victor Babeş" University of Medicine and Pharmacy, Piaţa Eftimie Murgu nr. 2, 300041, Timişoara, Timiş, Romania. Tel: +40 720060955, Fax: +40 256490626, e-mail: ancacimpean1972@yahoo.com

Key Words: Angiogenesis, chorioallantoic membrane, MCF-7, mesenchymal stem cells, vasculogenesis. rather than through angiogenesis, the process involving circulating bone marrow-derived endothelial precursors rather than mature endothelial cells (1).

Lately, mesenchymal stem cells (MSC) have generated substantial interest in the medical areas of transplant, regenerative medicine and cancer treatment because of their multi-potency and multi-functionality. MSC are a heterogeneous subset of stromal cells present in several tissues including bone marrow, bone, adipose tissue, skin, kidney, umbilical cord and placenta (2).

MSC are localized in the vascular niche in bone marrow, but also found as MSC-like cells around adult vessels and there is substantial evidence that they play a pivotal role in regulating blood vessel formation and function through multiple mechanisms such as vasculogenesis, arteriogenesis and angiogenesis (3).

While MSC differentiation towards vascular cell lineages and their incorporation into a growing vessel wall is rather ill-defined, their important contribution to promoting postnatal vascularization during ischemic myocardial tissue regeneration and tumor vasculogenesis is becoming increasingly recognized (4). It seems that hypoxia serves to enhance the differentiation of MSC towards the endothelial phenotype. Additionally, a revised understanding of MSCderived neovascularization contextualizes their behavior and utility as a hybrid endothelial-stromal cell type, with mixed characteristics of both populations (5).

Currently, the role of MSC in carcinogenesis is a matter of controversy. It has been reported that they favor tumor growth due to the immunosuppression they induce. Also, MSC could enhance tumor metastatic potential since they induce epithelial-to-mesenchymal transition (EMT). Other results prove that MSC inhibit tumorigenesis in epithelial precancerous lesions (2). In vivo and in vitro experiments reported, on the other hand, that adipose tissue derived stem cells favor tumor growth, increasing extracellular matrix deposition and vascularization (6). 
We have previously demonstrated, in an in vitro transmigration model, that both MCF-7 and VEGF alter the migration behavior of MSC, indicating a role of tumor cellderived VEGF to modulate the involvement of MSC in angiogenesis (7). Consecutively, we have proven that both MCF-7 and VEGF stimulate MSC to form capillary-like structures, indicating a role of tumor-derived VEGF in modulating their recruitment to breast cancer vasculogenesis (8).

CAM has since long been a favored system for the study of tumor angiogenesis and metastasis, because at this stage the chicken's immunocompetence system is not fully developed (9). The lack of a functional immune system during embryonic and fetal development permits the survival and development of cells from any species that tolerates the incubation temperature (10). Other advantages of this in vivo assay are the low cost, simplicity, reproducibility and reliability (9).

Using the CAM model, we demonstrated that MCF-7 seeded onto CAM recruit the mesenchymal cells from the CAM mesoderm and induce their differentiation towards myofibroblast-like or vasculogenic lineage, turning the mesoderm of the CAM into a surrogate tumor stroma (11). Further on, after the implantation onto CAM, hMSC induce vasculogenesis, by switching to an endothelial-like nonproliferative phenotype, but also by stimulating the chicken MSC from the mesoderm (cMSC) to acquire a vasculogenic and pericyte-like phenotype and to organize into cord/capillary like structures (CLS) (12).

All the experiments detailed above have evolved in a stepwise manner and have finally converged to the point of recreating an open scene for the in vivo interaction between hMSC and MCF-7 in order to put the lights on the intimate involvement of MSC in tumor vasculogenesis and angiogenesis.

\section{Materials and Methods}

hMSC cell culture. hMSC cells were purchased from Cellular Engineering Technologies Inc. (Coralville, IA, USA). Their molecular profile was previously documented by flow cytometry: CD 105+, CD90+, CD44+, CD29+, CD45-, CD14-. hMSC were culture expanded in $12 \mathrm{ml}$ of culture medium [low-glucose Dulbecco's modified Eagle medium (DMEM), containing 20\% Fetal Bovine Serum, 1\% mixture Penicillin/Streptomycin, supplemented with $25 \mathrm{ng} / \mathrm{ml}$ basic fibroblast growth factor] in T75 flasks. Cells were trypsinized when they reached a confluence of $70-80 \%$ and reseeded at $2-3 \times 10^{3} / \mathrm{cm}^{2}$. Medium change was performed twice a week. The cells from the passage 4 were used in experiments.

MCF-7 were purchased from Cell Lines Services (Eppelheim, Baden-Württemberg, Germany) and cultured at a density of $1 \times 10 \mathrm{E} 6$ cells/T75 plate in DMEM, containing $10 \%$ fetal bovine serum and $1 \%$ mixture Penicillin/Streptomycin. Medium change was carried out two times per week and the passage once a week, at a sub cultivation ratio of $1: 3$. Both cell lines were incubated at $37^{\circ} \mathrm{C}$, in a humidified atmosphere containing $5 \% \mathrm{CO}_{2}$.
CAM experimental model. Thirty-two fertilized chicken eggs were incubated at $37^{\circ} \mathrm{C}$. On day 3 of incubation, a window was cut in the eggs' shell and it was consecutively covered with Parafilm. The eggs were consecutively reincubated for further 7 days.

Cell implantation onto CAM. Two rings of silicone were placed onto the CAM surface of each egg, under sterile conditions, on day 10 of incubation (day 0 of the experiment). Depending on the ring inserts, the eggs were organized into 2 subsets: subset 1 (control eggs) - 8 eggs, subset 2 (experimental eggs) - 24 eggs. The eggs from subset 1 received $5 \mu \mathrm{l}$ of DMEM into the both rings. The eggs from subset 2 received a suspension of MCF-7 $(2 \times 10 \mathrm{E} 4$ cells/5 $\mu$ l DMEM) into one ring and a suspension of hMSC $(2 \times 10 \mathrm{E} 4$ cells $/ 5 \mu \mathrm{LMEM})$ into the other ring. The experiment was stopped 7 days later (day 7 of the experiment, day 17 of incubation). $5 \mathrm{ml}$ formalin $4 \%$ were instilled in ovo, onto the surface of the CAM. The viability of the eggs was assessed daily during the incubation using an Axio Zoom.V16 Zeiss stereomicroscope (Oberkochen, Baden-Württemberg, Germany) and only the eggs which maintained their viability until the end of the experiment were consecutively subject of the CAM examination.

Microscopic evaluation. In order to evaluate their morphology, CAMs were detached out of each egg and submerged for fixation in $4 \%$ formalin solution, for $48 \mathrm{~h}$, and then embedded in paraffin. Consecutively, 5 micrometers-thick sections were cut by the microtome and they were either stained with hematoxylin and eosin staining for the morphological evaluation, or they were processed for the evaluation of different immunohistochemical markers.

The incubation step of the immunohistochemical technique was performed for $15 \mathrm{~min}$, by using the following Bond ready-to-use primary antibodies for the specific human markers: ER (clone 6F11), PR (clone 16) and HER2 (clone CB 11) from Leica Biosystems Newcastle Ltd (Newcastle upon Tyne, Tyne and Wear, UK), CD45 (clone X16/99), smooth muscle actin (SMA) (clone $\alpha$ sm-1), $\beta$-catenin (clone 17C2), vimentin (clone V9) and CD105 (clone A-8) from Santa Cruz Biotechnology (Dallas, TX, USA), chick/human CD34 from Abbiotec (San Diego, CA, USA) and VEGF (clone 7G7) from ReliaTech (Wolfenbüttel, Lower Saxony, Germany). Bond Polymer Refine Detection System was used for visualization of the simple immunostainings. In the case of double immunostainings (CD34/SMA, VEGF/CD34 and $\beta$-catenin/vimentin), Bond Polymer Refine Red Detection System (Leica Biosystems) was used for visualization. The chromogen dihidrochlorid-3, 3-diamine-benzidine was applied for $10 \mathrm{~min}$. Hematoxylin applied for $5 \mathrm{~min}$, was used as counterstain. The entire procedure was performed with the Leica Bond-Max autostainer (Leica Biosystems).

The intensity of the immunohistochemical reaction was classified as it follows: negative $(-)$, low $(+)$, moderate $(++)$, high $(+++)$. The morphological and immunohistochemical slides were scanned using the Pannoramic Desk scanner from3D HISTECH (Budapest, Hungary) and the images werevisualized and recorded using the Pannoramic Viewer Software.

\section{Results}

The evaluation of viability among the subsets of eggs at the end of the experiment returned the following values: subset 1 (control eggs) - 7 viable eggs out of 8 , subset 2 (experimental eggs) - 18 viable eggs out of 24 . The viable eggs were processed and, consecutively, microscopically evaluated. 

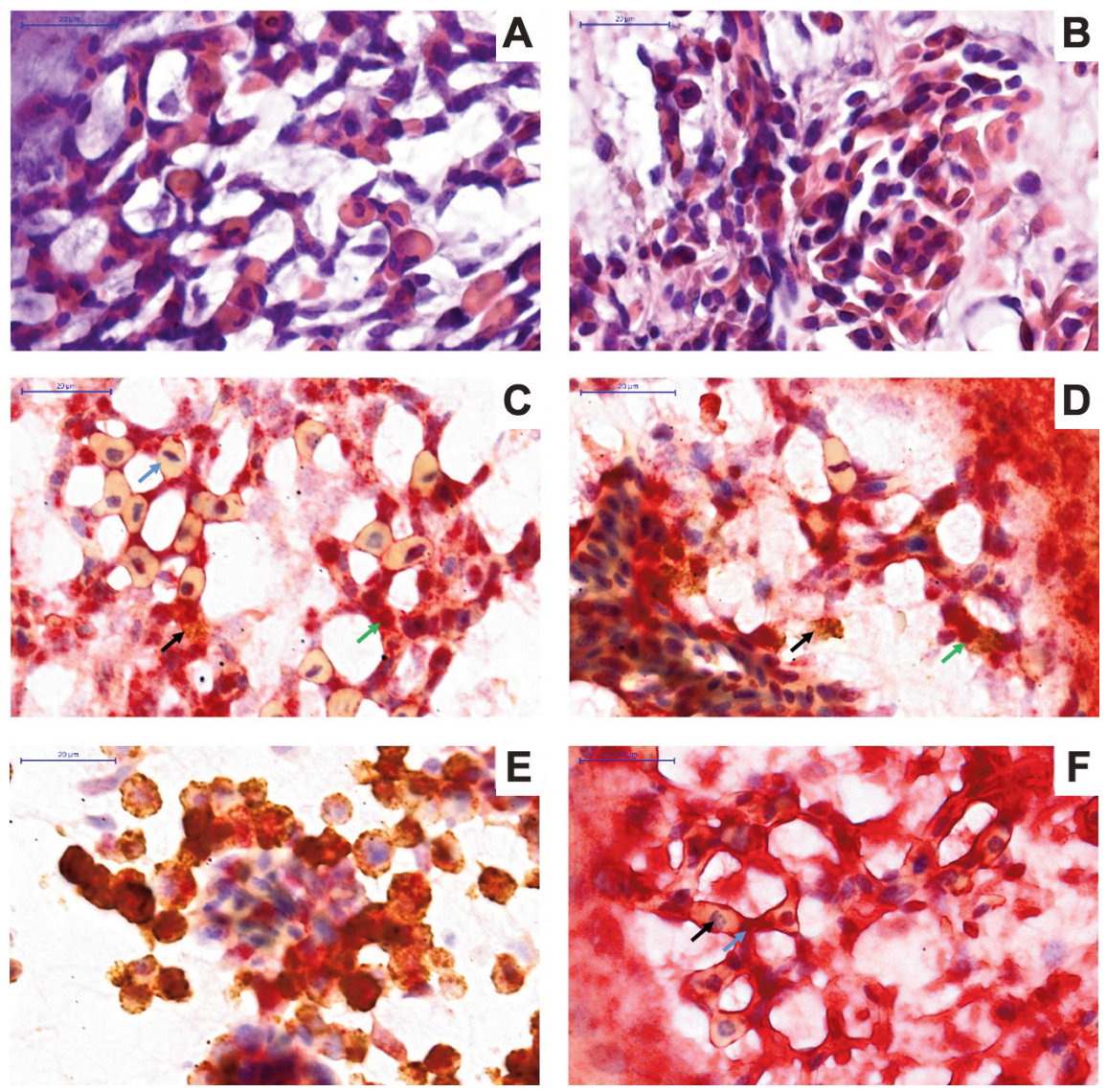

Figure 1. Morphological and immunohistochemical features of CAM after seeding hMSC and MCF-7. a) Hematoxylin-eosin staining: Network of $C L S$ at the level of the CAM mesoderm, in the proximity of the chorionic epithelium, containing a puzzle of endothelial-like cells and hematopoietic cells; b) Hematoxylin-eosin staining: Area of hematopoiesis with incipient signs of vasculogenesis in the proximity; c) VEGF expression (brown)/CD34 expression (red): CLS formed by VEGF $\pm C D 34++$ cMSC (green arrow) include VEGF+/CD34+ hematopoietic cells (blue arrow) and rarely VEGF $+++/ C D 34+M C F-7$ (black arrow); $d$ ) VEGF expression (brown)/CD34 expression (red): VEGF $+++/ C D 34+M C F-7$ in contact with CLS (black arrow) and included in the CLS (green arrow); e) VEGF expression (brown)/CD34 expression (red): VEGF +++/CD34+ MCF-7 form a tubule-like structure around a cluster of VEGF+/CD34+ hematopoietic cells; f) CD34 expression (brown)/SMA expression (red): cMSC forming CLS express both SMA (+++) and CD34 (+), while the hematopoietic cells express only CD34 (+). Magnification 1,000x.

Morphological evaluation. In the control eggs, a tree branching system of readily formed functional vessels was visible in the mesoderm. The mesoderm presented a dense cellularity, while the mesenchymal morphology was not so prominent. In addition, a developing vascular sinus was identified in the mesoderm, in the intimate proximity of the chorionic epithelium. The structure could be characterized as a string of luminal structures with rare blood cells, separated by cytoplasmic pillars, presenting a tendency to confluence. No morphological features of angiogenesis or vasculogenesis were identified.

Seeding hMSC and MCF-7 onto CAM induced important morphological changes of the substrate. The connective tissue from the mesoderm developed an embryonal mesenchymal morphology, with star-shaped cells forming a network through their thin and long prolongations.
At the level of their seeding site, $\mathrm{MCF}-7$ crossed the chorionic epithelium and entered the mesoderm, where they could be identified mainly as solitary cells. Seldom, they appeared organized in small clusters, especially in the proximity of the CAM vessels. Rarely MCF-7 proved a tendency for tumor mass organization, with surrounding mesenchymal and inflammatory reaction. They also entered the lumen of the large vessels from the mesoderm, but in a lesser degree. MCF-7 promoted vasculogenesis in the mesoderm, by inducing the local cMSC to form networks of CLS, mainly in the proximity of the chorionic epithelium. These networks extended towards and inside of the chorionic epithelium. CLS demonstrated a tendency to connect to the existing vessels from the mesoderm. At the level of these networks, vasculogenesis and hematopoiesis were generally concomitant and co-localized. The CLS contained a puzzle of endothelial- 
like cells and hematopoietic cells - primitive nucleated red blood cells (Figure 1a). CLS with no hematopoietic cells, but also with extra-luminal hematopoiesis, with vascular lumen development around the existing primitive nucleated red blood cells may be documented (Figure 1b). The mechanism of vascular lumen formation at the level of CLS was represented by both cell and cord hollowing.

MCF-7 also stimulated angiogenesis, as a tree-branching system developing out of the existing vessels could be noticed in the deeper mesoderm. We identified a concentration of tumor cells, solitary or organized in clusters, in the intimate proximity of the hotspots of vasculogenesis and angiogenesis.

In the proximity of the hMSC seeding site, hMSC crossed the chorionic epithelium and invaded the mesoderm, the process took place in an organized manner, most of the cells presented a common axis of migration. MCF-7 were strongly attracted by hMSC, as a large number of them migrated towards the implantation site of hMSC.

Comparing with the MCF-7 implantation site, reduced CLS formation, involving vasculogenesis and hematopoiesis, was identified, especially under the chorionic epithelium and extending to it. CLS formed rarely a network, but they proved a tendency to connect to the existing vessels of the mesoderm, too. On the other hand, the angiogenic response was intense, the microvascular density being significant in the hMSC implantation zone.

Immunohistochemical evaluation. In the control group, only SMA was positive onto CAM, presenting a high expression in the proximity of the large vessels. All the other markers were not expressed onto CAM.

We describe below the immunohistochemical profile of the experimental eggs.

VEGF expression. hMSC presented a moderate positive VEGF expression with granular pattern, documented both in the chorionic epithelium and in the mesoderm at their implantation site, while cMSC presented a low, inconstant VEGF expression. VEGF expression in the endothelial-like cMSC forming CLS was similar to the expression in the other cMSC. Based on this pattern of expression, we also identified hMSC at the site of MCF-7 implantation, which demonstrates that hMSC were attracted by MCF-7.

MCF-7 had a moderate-high VEGF expression. They were identified in the chorionic epithelium and the CAM mesoderm. The VEGF-positive MCF-7 could be identified all over the mesoderm, those with high VEGF expression showing an affinity for the blood vessels and the CLS. A small amount of these highly positive MCF-7 was noticed in the large vessels' wall or in their lumen. The hematopoietic cells forming in the CLS had a low VEGF expression. The expression was also low, but inconstant for the blood cells from the already formed vessels.
CD34 expression. hMSC had a moderate-high CD34 expression in the chorionic epithelium and the mesoderm. The cMSC population had generally a low-moderate CD34 expression, but it was moderate-high in the endothelial-like cMSC from the areas of vasculogenesis. The endothelial cells of the large vessels also had a low-moderate CD34 expression. MCF-7 also presented a low-moderate, inconstant CD34 expression. The blood cells had a low CD34 expression both in the hematopoietic areas and in the large vessels.

$V E G F / C D 34$ expression. The two markers were co-expressed by MCF-7, although there was a subpopulation which expressed only VEGF. VEGF+++/CD34+ and VEGF+++ tumor cells could be identified in the proximity of the blood vessels and CLS, establishing direct cell contact or through prolongations. Rarely these cells could be noticed in the structure of the CLS network (Figure 1c and d). Interestingly, the tumor cells expressing VEGF, or both markers, were organized in clusters, especially in the proximity of the vessels or the vasculogenesis/angiogenesis sites, and, sometimes, proved a tendency to form cord-like and even tubule-like structures, in some instances enveloping VEGF+/CD34+ hematopoietic cells (Figure 1e). The coexpression was also visible in the hMSC from the chorionic epithelium and the mesoderm. The cMSC cells, mainly the endothelial-like cMSC forming the CLS, co-expressed CD34 and VEGF, too, but inconstantly (Figure 1c and d).

SMA expression. SMA was intensely expressed by hMSC crossing the chorionic epithelium, but also by those entering the mesoderm. cMSC had an intense SMA expression in the sites of hMSC and MCF-7 implantation, especially in the perivascular areas; a similar pattern of expression was visible in the endothelial-like cMSC, at the level of CLS. In the other mesodermal areas the expression was low-moderate. The cells from the wall of the large vessels also had a high SMA expression. MCF-7, mainly those located in the proximity of MCF-7 and hMSC seeding sites had a lowmoderate inconstant expression.

CD34/SMA expression. Using the double immunostaining method, we identified the co-expression of CD34 and SMA in hMSC, but also in the endothelial-like cMSC especially in those involved in CLS formation (Figure 1f). MCF-7 were identified in 3 variants: CD34+, SMA+, CD34+/SMA+.

CD105 expression. hMSC had a low, inconstant CD105 expression at the level of the chorionic epithelium and in the mesoderm. MCF-7 also had a low, inconstant CD105 expression. The other cells did not express CD105.

CD45 expression. None of the hematopoietic cells, either from the existing vessels or CLS, and no other cells expressed CD45. 
$\beta$-catenin/vimentin expression. MCF-7 presented a lowmoderate expression for $\beta$-catenin at the level of the chorionic epithelium, but this expression was down-regulated as they entered the mesoderm, where the expression was low. Interestingly, the pattern of expression for the tumor cells from the mesoderm was both membrane and cytoplasmic. On the other hand, MCF-7 did not express vimentin neither at the level of the chorionic epithelium, nor in the mesoderm.

$\beta$-catenin also presented a low expression in the endothelial cells from the existing vessels and, inconstantly, in the MSC population from the area of MCF-7 implantation. hMSC presented a moderate vimentin expression at the level of the chorionic epithelium, but they lost this expression as they entered the mesoderm.

ER, PR, HER 2 expression. MCF-7 and the other cells present onto CAM were immunohistochemically negative for ER, PR and HER2.

\section{Discussion}

The co-implantation of MSC and MCF-7 onto CAM represents a cornerstone for the in vivo analysis of vasculogenesis and angiogenesis. By implanting the two cell lines onto CAM, we have established a human-avian xenotransplant and have proven again that hMSC, but also $\mathrm{MCF}-7$, engraft and function across the species barriers, as was documented before (13).

The current experiment puts into light the behavior of both MSC and MCF-7 after being seeded onto CAM, in an in vivo setting which facilitates their interaction. In addition, it reveals their involvement in vasculogenesis and angiogenesis, but also their ability to change the scene of their interaction.

The implantation of MCF-7 and hMSC onto CAM seems to have reset the mesoderm to an earlier stage of development, as its connective tissue went back to the embryonal mesenchymal morphology, with star-shaped cells forming a network through their thin and long prolongations. These features were not visible in the control eggs, where the connective tissue from the CAM mesoderm continued the evolution toward a more differentiated estate.

Another argument for this reset is represented by the hotspots of angiogenesis and vasculogenesis associated with hematopoiesis generated by hMSC and MCF-7 in the CAM mesoderm. The identification of such features on the day 17 of incubation is uncommon for the CAM. After day 9, the CAM vasculature is fully established and allows investigation under conditions without the background angiogenesis (14).

The explanation for this re-activation of vasculogenesis/ hematopoiesis and angiogenesis onto CAM is that any tissue growth (developmental, repair-related or malignant) requires new vasculature. Tissue stem cells have the ability to form vasculature de novo when needed. Which cells would produce a new piece of tissue or its vasculature does not have to be predetermined during embryogenesis but later, when and where the growth is to take place (1). The new element brought by the current study is the influence of the two co-implanted cell lines on the balance between angiogenesis and vasculogenesis.

MCF-7 stimulated more vasculogenesis than angiogenesis and this finding supports a previous study which demonstrated that tumors forming spontaneously from a single cell, which are more representative of human tumors, display a more consistent requirement for utilizing vasculogenesis. Moreover, advanced tumors have been shown to preferentially utilize vasculogenesis to accommodate the increasing requirement for oxygen and nutrients (4).

On the other hand, hMSC induced a more intense angiogenic response, but a reduced vasculogenic reaction. Interestingly, when implanted alone onto CAM, hMSC determined an intense vasculogenic reaction, in addition to the angiogenic response (12), which means that MCF-7 have the ability to modulate the involvement of hMSC in neovessel formation. hMSC were demonstrated to enhance the de novo formation of stable vasculature by endothelial colony-forming cells in surrogate models of vasculogenesis in vitro and in vivo (3), in our study cMSC being the source for the endothelial colony-forming cells. MSC do not only promote vasculogenesis, but also angiogenesis from primary human endothelial precursors in vitro (3). In addition, human MSC from umbilical cord blood demonstrated their ability to directly self-organize forming new functional vasculature connected with the host circulatory system once implanted in mice (3), although this could not be demonstrated after implanting hMSC in the chick embryo CAM, probably due to mammal-avian species barrier.

Onto CAM, in the presence of hMSC, MCF-7 were identified mostly solitary and they were strongly attracted by hMSC. An increasing number of evidence has shown that MSC in primary tumors can differentiate in active components of the tumor microenvironment such as cancerassociated fibroblasts, which are known to play a key role in tumor progression, neo-angiogenesis, EMT and metastatic spread (15). In vitro migration studies revealed an approximately 4-fold increase in migration of MCF-7 cells cultured in the presence of hMSC under basal and stimulated chemotaxis conditions, suggesting the involvement of hMSC in the promotion of a metastatic phenotype (16). More than $80 \%$ of MCF-7 co-cultured with MSC were present as single cells lying close together without any evidence of direct cellcell contacts, while the expression of the main epithelial intercellular adhesion molecules was down-regulated in MCF-7 (17). The dissociation of tumor cells from one another might be due to hypoxic stress and starvation and the loss of attachment to other cells could facilitate their dissemination by breaking tumor tissue into small cell 
clusters or single cells that could be carried away by the blood flow (18).

Interestingly, hMSC crossed the chorionic epithelium and invaded the mesoderm in an organized manner, most of the cells presenting a common axis of migration. Endothelial cells also exhibit the capacity for coordinated (collective) migration, identified clusters of genes having prominent and specific effects on various aspects of motility, such as activity (speed) and coordination with adjacent cells (19). hMSC could also share with endothelial cells the motility response to external gradient of growth factors, in particular to VEGF, or the autocrine chemotaxis (19), as VEGFpositive MCF-7 could be identified concentrated in the proximity of their implantation site, they themselves also expressing VEGF.

The alteration in the cellular functionality of tumor cell populations and MSC within the tumor microenvironment contributes to tumor heterogeneity and suggests a process of mutual cellular adaptation whereby MSC acquire tumor cellspecific markers and vice versa. Co-culture of various human cell populations with different individual MSC populations was accompanied by exchange of biological material via different mechanisms, including exosomes and formation of nanotubes (20).

Seeding both MCF-7 and hMSC onto CAM had an important impact on the molecular profile of both cells. Onto $\mathrm{CAM}$, in the presence of $\mathrm{hMSC}, \mathrm{MCF}-7$ presented a triple negative phenotype, but this aspect was also identified when MCF-7 were seeded alone onto CAM (11). The result demonstrates that hMSC do not change this status acquired by MCF-7 onto CAM.

MCF-7 have developed a moderate-high VEGF expression onto CAM, although they presented only a moderately positive status when implanted alone (11), while, normally, the cell line is characterized by secretion of immunodetectable, but low levels of VEGF-A (21) and a poor angiogenic potential (22). Our results clearly demonstrate that CAM activates the angiogenic, but also the vasculogenic potential of MCF-7, while hMSC supplementary stimulate it. Highly VEGF-positive MCF-7 were identified in the proximity of the vessels and CLS, few of them being present in the wall and even the lumen of the CAM vessels, but also inserted in the CLS network. It seems that this behavior correlates with VEGF expression, because it has been demonstrated that the patients with vascular invasion exhibited significantly higher VEGF expression rates compared to patients with no vascular invasion in breast cancer (23).

MCF-7 also presented a low-moderate, inconstant CD34 expression, although in vitro, MCF-7 do not express CD34 (24). CD34 labeling of melanoma cells, based on the epithelioid shape of the tumor cells, was previously described. Multiple cells stained in grapelike clusters, while other elements formed tubular structures consistent with vessels (25).
In addition, the gastric adenocarcinoma aggressive tumor cells generating a tubular-type of vascular mimicry (VM), rather than a patterned matrix type, and mosaic vessels, were found to express CD31 or CD34, thus angiogenic and vasculogenic markers, probably due to genetically deregulation (26). Although the classical pattern in VM morphology is PASpositive and CD34-negative mature tumor vessels (27), we also noticed CD34-positive/VEGF-positive tumor cells organized in clusters or cords. In some instances, they were forming tubular structures, thus initiating the process of VM.

Since its initial identification in human cutaneous and uveal melanomas, VM has been observed in most malignant tumors, including the tumors of the breast (28), and it has been recognized that $\mathrm{VM}$ is not only involved in proliferation and metastatic potential but is also associated with a poor patient prognosis (27). Triple-negative breast cancer, the breast cancer subtype with the most pessimistic survival rates, exhibits increased VM compared to other subtypes. In addition, MCF-7 breast cancer cells suffering EMT are able to form VM channels in 3D culture medium (28). We previously demonstrated that after being seeded onto CAM, MCF-7 changed their molecular profile and acquired a more aggressive status. They became triplenegative and entered EMT, as they started expressing vimentin, concomitant with the loss of $\beta$-catenin and CK18 (11). When co-implanted with hMSC, MCF-7 did not express vimentin, but they had a low-moderate inconstant SMA expression. SMA and vimentin are not usually expressed in MCF-7 epithelial cells (29), but MDA-MB-231 breast cancer cells, which are considered to have underwent EMT, are positive for mesenchymal markers such as vimentin and SMA (30), so we could suppose that SMA expression in MCF-7 could translate a particular form of EMT, modulated by hMSC.

Hypoxia also induces VM formation in breast cancer and is considered the most potent factor affecting the process (28). Hypoxia stimulates VEGF expression through hypoxiainducing factors pathway (28) and VEGFA has been considered to play a crucial role in the formation of $\mathrm{VM}$ (27). The binding of VEGFA to its ligand and activation of VEGFR1 and/or VEGFR2 results in cell proliferation, angiogenesis and VM formation in tumors (27). The pattern of VEGF expression in MCF-7, but also the hotspots of vasculogenesis and angiogenesis the tumor cells generate onto CAM, suggest the involvement of hypoxia and so, taking all this information together, we can conclude that onto CAM, MCF-7 are eligible candidates for VM.

MCF-7 had a low, inconstant CD105 expression and the explanation could be that CD105 induction in the tumor cells, previously observed at both the protein and mRNA levels, is the result of an exchange of proteins and/or transcriptional regulators and/or mRNAs between MSC and co-cultured tumor cells (20). It is also interesting to mention 
that doxorubicin resistant-MCF-7 and MDA-MB-231 express CD105, while the wild-type MCF-7 human breast cancer cell line does not express it (31). Similar to MCF-7 seeded onto CAM, doxorubicin resistant-MCF-7 lack ER and PR (32), while MDA-MB-231 express mesenchymal markers, thus proving EMT (30). Accordingly, we may consider that CD105 expression in MCF-7 may be linked to ER and PR loss, but also to EMT, thus to acquisition of a more aggressive status by the tumor cells onto CAM.

MCF-7 presented a low-moderate $\beta$-catenin expression in the chorionic epithelium, but this expression was downregulated as they entered the mesoderm, the pattern being both membrane and cytoplasmic. $\beta$-catenin is a subunit of the cadherin adhesion complex and mainly localizes to the cell membrane (33). In MCF-7, $\beta$-catenin is mostly expressed at the same level (34), but a shift in subcellular localization of $\beta$-catenin may be possible (35). Reduced membranous $\beta$-catenin expression combined with accumulation of free cytoplasmic and nuclear pools of $\beta$ catenin transcriptionally regulates target genes that affect tumor invasion and/or metastasis (33). $\beta$-catenin also has a dual role in the EMT; it enhances cell-cell adhesion when bound to cadherin complexes in adherens junctions and also functions as a transcriptional coactivator upon entry into the nucleus, nuclear import of $\beta$-catenin being an important player in EMT (34). In addition, the high cytoplasmic expression of $\beta$-catenin is surprisingly associated with ERand PR-negative status (35), a status also acquired by MCF7 onto CAM. So, we may conclude that the pattern of $\beta$ catenin expression in MCF-7 seeded onto CAM is linked to the hormone receptor negative status and EMT.

MSC-like cells have been linked to the angiogenic switch process by the secretion of proangiogenic factors, such as VEGF, as well as by contributing to the recruitment of circulating vascular progenitor cells (3). In our experiment, hMSC presented a moderate positive VEGF expression and clearly stimulated cMSC to form CLS. On the other hand, growth factors, such as VEGF, have been found to effectively mediate MSC homing to tumor microenvironment (15), so that the hMSC migration towards the MCF-7 implantation area must have been stimulated by the tumor VEGF.

The VEGF lack of expression in cMSC may be related to the commitment of these cells towards other lineages, while the VEGF re-expression in some cMSC, which seems to have been stimulated by both hMSC and MCF-7, proves the plasticity of these cells and the ability to regain their stemness. The fact that VEGF expression in cMSC forming CLS was low, inconstant and similar to that of the other neighboring cMSC demonstrates that CLS formation does not require VEGF expression in cMSC. Anyway, the exogenous VEGF, secreted by hMSC and MCF-7, must have been involved in the process of vasculogenesis, as VEGF is known to regulate MSC mobilization and recruitment to sites of neovascularization, as well as directing the differentiation of MSC to a vascular cell fate (4).

When co-implanted with MCF-7, hMSC developed a moderate-high CD34 expression onto CAM, while cMSC presented generally a low-moderate CD34 expression, with a moderate-high intensity in the areas of vasculogenesis/ angiogenesis. Surprisingly, when implanted alone hMSC had an isolated moderate positive CD34 expression, while cMSC had a constantly low expression, the expression being restricted to the vasculogenic areas (12). These data suggest that CD34 expression is up-regulated by MCF-7 in both hMSC and cMSC.

Nevertheless, it is well established that cultured colonies of MSC do not express CD34 (36). Some groups have reported the direct derivation of $\mathrm{CD} 34+$ primitive MSC from human embryonic stem cells (hESC), while others described the emergence of a multipotent MSC precursor, the mesenchymal-angioblast, from hESC-derived CD34+ cells in a stepwise differentiation system (37). Thus, it seems that MSC derive from a CD34+ precursor, while their negativity for CD34 is likely a cell culture-induced phenomenon, not indicative of their actual in vivo status (38).

CD34 expression in MSC may indicate the acquisition of an endothelial/hematopoietic-like phenotype, CD34 being expressed on endothelial cells as well as on hematopoietic stem and progenitor cells in the embryo and adult (39). Furthermore, the CD34+ adventitial progenitor cells, which are de facto MSC, were considered "vascular stem cells", because they were capable of differentiating into mature hematopoietic cells, endothelial cells and macrophages (38).

SMA was intensely expressed by hMSC, while cMSC had an intense SMA expression only in the sites of hMSC and MCF-7 implantation, especially in the perivascular areas and at the level of CLS, in the other mesodermal areas the expression being low-moderate.

Several studies have demonstrated that MSC express abundant SMA, suggesting an enhanced vascular SMC-like differentiation (4). Although MSC possess inherent vascular SMC characteristics (4), their differentiation toward vascular $\mathrm{SMC} /$ pericyte lineages is presumably induced by direct contact with endothelial tubes (40).

There are studies demonstrating the existence of common progenitors for endothelial cells and smooth muscle cells (41). Using the double immunostaining method, we identified the co-expression of CD34 and SMA in the cMSC involved in CLS formation. As embryonic vascular progenitor cells can differentiate into endothelial or smooth muscle cells, in response to VEGF (4), we may issue that cMSC behave like the embryonic vascular progenitor cells, probably in response to VEGF, too.

The SMA-positive c/hMSC may also act as cancerassociated myofibroblasts, as MSC have been shown to target the stroma environment of epithelial tumors, the tumor environment being able to activate MSC to myofibroblasts. 
Recent studies evaluating the interaction between MSC and epithelial tumor cells in vivo and in vitro indicate that acquisition of the myofibroblast phenotype by MSC (42).

CD105 expression was surprisingly low and inconstant in the hMSC from the chorionic epithelium and the mesoderm. This result is consistent with our previous findings after the implantation of hMSC alone onto CAM (12) and may be related to the in vitro-in vivo transition of the cells. Interestingly, MSC from the adipose tissue have been shown to express CD105 at low levels when freshly isolated, but become increasingly CD105+ upon culture passages. In any event, the data from adipose MSC suggest that CD105 may not be a useful marker for in vivo MSC detection (38). In addition, serum-free cultured hMSC display a significantly lower amount of CD105 protein compared to cells grown in presence of serum (43). The expression of CD105 is considered to vary hugely between culture conditions and laboratories. Anyway, the CD105+MSC and CD105-MSC have similar stemness and in vitro differentiation potential to become osteoblasts, chondroblasts and adipocytes, while the CD105-MSC have a stronger capacity of immune modulation compared to CD105+ MSC (44).

hMSC presented a moderate vimentin expression at the level of the chorionic epithelium, but they lost this expression as they entered the mesoderm. On the other hand, cMSC were all negative. Vimentin lack of expression in the hMSC residing in the mesoderm may be linked to a commitment of these cells towards a particular lineage. It is not known whether vimentin intermediate filaments (IFs) undergo active remodeling within all lineage commitments (45), but generally a given cell type may express successively different classes of IFs during its differentiation; for instance, myoblasts synthesize exclusively vimentin and switch to a predominant expression of desmin when they fuse into myotubes (46). In addition, the quantitative expression of IFs may be altered during cell differentiation, $3 \mathrm{~T} 3$ preadipocytes exhibiting a striking decrease in vimentin, actin and tubulin during adipose differentiation (46).

Another hypothesis regarding the vimentin lack of expression is that the hMSC mimic the host, as xenotransplantation of human MSC in the rabbit gravid uterus resulted in differentiation and production of human liver cells in the liver of rabbit infants (47). On the other hand, the antibody V9 was shown to react with the vimentin of various mammalian species, but it did not detect vimentin in mouse cells or chicken fibroblasts (48), so explaining why cMSC were negative. These findings suggest that IFs of hMSC could mimic the structure of those from cMSC, thus explaining the "escape" of hMSC from expressing vimentin.

During the cellular interactions between MSC and tumor cells, a variety of functional changes were observed, particularly the acquisition of multiple epithelial cell-like properties by MSC (20). Accordingly, MSC population residing in the area of MCF-7 implantation and presenting a low $\beta$ - catenin expression may have gained this molecule through cellular interactions with MCF-7. $\beta$-catenin expression in MSC may also be interpreted from another perspective. $\beta$-catenin expression is able to up-regulate Oct 4 expression and interact with Nanog and thus promote self-renewal. So, an enhance in $\beta$-catenin expression in MSC facilitates the maintenance of MSC self-renewal (49). The morphological features and the molecular profiles acquired by hMSC and MCF-7 onto CAM indicate an alteration in the cellular functionality of both the tumor cells and MSC within the tumor microenvironment, which contributes to tumor heterogeneity and suggests a process of mutual cellular adaptation, whereby MSC acquire tumor cell-specific markers and vice versa.

The advantages of the current study are obvious. We managed to create a surrogate tumor microenvironment which allowed us to analyze the interaction between the tumor cells and MSC, but also the result of this interaction, translated through complex phenotypical changes, present in both the tumor cells and MSC. In addition, we had access to the intimate involvement of the MSC in the processes of tumor vasculogenesis and angiogenesis. The disadvantage of this study is the avian origin of the experimental model, which may have restricted some tissue processes due to the human-avian barrier. On the other hand, the results of this study need to be confirmed on mammalian experimental models in order to prove that they cross the species barrier.

\section{Conclusion}

hMSC and MCF-7 develop a complex interaction with each other but also with CAM, which results into morphological and molecular changes, as follows: i) $\mathrm{MCF}-7$ cells prove their affinity for hMSC, suffer a prometastatic arrangement, acquire a proangiogenic/provasculogenic molecular profile, enter a particular form of EMT and initiate the VM process; ii) hMSC migrate towards $\mathrm{MCF}-7$ seeding site, involve in the tumor stroma formation and switch to a vascular precursor phenotype; iii) CAM represents a real niche for both hMSC and MCF-7, which allows them to survive and cooperate; as a result of this hosting, CAM undergoes a major reset to an earlier stage, as it generates hotspots of angiogenesis, vasculogenesis and hematopoiesis, the processes being modulated by the both cell lines.

The hallmark of the current study is the establishment of a veritable in vivo experimental model of MSC involvement in tumor vasculogenesis and angiogenesis, which allows future experiments for revealing so many unknown molecular mechanisms these cells possess.

\section{Conflicts of Interest}

The Authors declare there are no conflicts of interest associated with this publication. 


\section{Authors' Contributions}

ŞC and AMC designed the study. RP performed the cell culture. ŞC managed the CAM experimental model and A-RC the morphological and immunohistochemical stainings. ŞC, AMC, SS and MR analyzed the data. ŞC wrote the draft. All the Authors revised the manuscript.

\section{Acknowledgements}

This work was supported by the project 4POSTDOC/1318/31.01.2020 from the "Victor Babeş" University of Medicine and Pharmacy, Timişoara, Romania.

\section{References}

1 Witkiewicz H, Oh P and Schnitzer JE: I. Embryonal vasculature formation recapitulated in transgenic mammary tumor spheroids implanted pseudo-orthotopicly into mouse dorsal skin fold: the organoblasts concept. F1000Research 2: 8, 2013. PMID: 24555024. DOI: 10.12688/f1000research.2-9.v2

2 Bruna F, Arango-Rodríguez M, Plaza A, Espinoza I and Conget $\mathrm{P}$ : The administration of multipotent stromal cells at precancerous stage precludes tumor growth and epithelial dedifferentiation of oral squamous cell carcinoma. Stem Cell Res 18: 5-13, 2017. PMID: 27939557. DOI: 10.1016/j.scr.2016.11.016

3 Watt SM, Gullo F, van der Garde M, Markeson D, Camicia R, Khoo CP and Zwaginga JJ: The angiogenic properties of mesenchymal stem/stromal cells and their therapeutic potential. Br Med Bull 108: 25-53, 2013. PMID: 24152971. DOI: $10.1093 / \mathrm{bmb} / \mathrm{ldt} 031$

4 Ball SG, Shuttleworth CA and Kielty CM: Mesenchymal stem cells and neovascularization: role of platelet-derived growth factor receptors. J Cell Mol Med 11: 1012-1030, 2007. PMID: 17979880. DOI: 10.1111/j.1582-4934.2007.00120.x

5 Rytlewski JA, Aldon MA, Lewis EW and Suggs LJ: Mechanisms of tubulogenesis and endothelial phenotype expression by MSCs. Microvasc Res 99: 26-35, 2015. PMID: 25711526. DOI: $10.1016 /$ j.mvr.2015.02.005

6 Bielli A, Scioli MG, Gentile P, Agostinelli S, Tarquini C, Cervelli V and Orlandi A: Adult adipose-derived stem cells and breast cancer: a controversial relationship. Springerplus 3: 345, 2014. PMID: 25089245. DOI: 10.1186/2193-1801-3-345

7 Comşa Șerban, Ciuculescu F, Henschler R and Raica M: MCF7MSC co-culture assay: approach to assess the co-operation between MCF-7s and MSCs in tumor-induced angiogenesis. Rom J Morphol Embryol 52: 1071-1076, 2011. PMID: 22119827.

8 Comşa Ş, Ciuculescu F and Raica M: Mesenchymal stem celltumor cell cooperation in breast cancer vasculogenesis. Mol Med Rep 5: 1175-1180, 2012. PMID: 22367291. DOI: 10.3892/ mmr.2012.796

9 Ribatti D, Nico B, Vacca A, Roncali L, Burri PH and Djonov V: Chorioallantoic membrane capillary bed: A useful target for studying angiogenesis and anti-angiogenesis in vivo. Anat Rec 264: 317-324, 2001. PMID: 11745087. DOI: 10.1002/ar.10021

10 Boulland J, Halasi G, Kasumacic N and Glover JC: Xenotransplantation of human stem cells into the chicken embryo. J Vis $\operatorname{Exp}$ (41): 2071, 2010. PMID: 20644515. DOI: 10.3791/2071
11 Comşa Ş, Popescu R, Avram Ş, Ceaușu R, Cîmpean A and Raica $\mathrm{M}$ : Bevacizumab modulation of the interaction between the MCF-7 cell line and the chick embryo chorioallantoic membrane. In Vivo 31: 199-204, 2017. PMID: 28358700. DOI: 10.21873/invivo.11045

12 Comşa Ş, Ceaușu RA, Popescu R, Cîmpean AM and Raica M: The human mesenchymal stem cells and the chick embryo chorioallantoic membrane: The key and the lock in revealing vasculogenesis. In Vivo 31: 1139-1144, 2017. PMID: 29102936. DOI: 10.21873 /invivo.11180

13 Li J, Ezzelarab MB and Cooper DKC: Do mesenchymal stem cells function across species barriers? Relevance for xenotransplantation. Xenotransplantation 19: 273-285, 2012. PMID: 22978461. DOI: 10.1111/xen.12000

14 Nowak-Sliwinska P, Alitalo K, Allen E, Anisimov A, Aplin AC, Auerbach R, Augustin HG, Bates DO, van Beijnum JR, Bender RHF, Bergers G, Bikfalvi A, Bischoff J, Böck BC, Brooks PC, Bussolino F, Cakir B, Carmeliet P, Castranova D, Cimpean AM, Cleaver O, Coukos G, Davis GE, De Palma M, Dimberg A, Dings RPM, Djonov V, Dudley AC, Dufton NP, Fendt SM, Ferrara N, Fruttiger M, Fukumura D, Ghesquière B, Gong Y, Griffin RJ, Harris AL, Hughes CCW, Hultgren NW, IruelaArispe ML, Irving M, Jain RK, Kalluri R, Kalucka J, Kerbel RS, Kitajewski J, Klaassen I, Kleinmann HK, Koolwijk P, Kuczynski E, Kwak BR, Marien K, Melero-Martin JM, Munn LL, Nicosia RF, Noel A, Nurro J, Olsson AK, Petrova TV, Pietras K, Pili R, Pollard JW, Post MJ, Quax PHA, Rabinovich GA, Raica M, Randi AM, Ribatti D, Ruegg C, Schlingemann RO, SchulteMerker S, Smith LEH, Song JW, Stacker SA, Stalin J, Stratman AN, Van de Velde M, van Hinsbergh VWM, Vermeulen PB, Waltenberger J, Weinstein BM, Xin H, Yetkin-Arik B, YlaHerttuala S, Yoder MC and Griffioen AW: Consensus guidelines for the use and interpretation of angiogenesis assays. Angiogenesis 21: 425-532, 2018. PMID: 29766399. DOI: 10.1007/s10456-018-9613-x

15 Camorani S, Hill BS, Fontanella R, Greco A, Gramanzini M, Auletta L, Gargiulo S, Albanese S, Lucarelli E and Cerchia L: Inhibition of bone marrow-derived mesenchymal stem cells homing towards triple-negative breast cancer microenvironment using an anti-PDGFR $\beta$ aptamer. Theranostics 7: 3595-3607, 2017. PMID: 28912898. DOI: 10.7150/thno.18974

16 Rhodes LV, Antoon JW, Muir SE, Elliott S, Beckman BS and Burow ME: Effects of human mesenchymal stem cells on ERpositive human breast carcinoma cells mediated through ERSDF-1/CXCR4 crosstalk. Mol Cancer 9: 295, 2010. PMID: 21087507. DOI: 10.1186/1476-4598-9-295

17 Hombauer $\mathrm{H}$ and Minguell J: Selective interactions between epithelial tumour cells and bone marrow mesenchymal stem cells. Br J Cancer 82: 1290-1296, 2000. PMID: 10755403. DOI: 10.1054/bjoc.1999.1093

18 Witkiewicz H, Oh P and Schnitzer JE: Capsular vaso-mimicry formed by transgenic mammary tumor spheroids implanted ectopically into mouse dorsal skin fold: implications for cellular mechanisms of metastasis. F1000Research 2: 9, 2013. PMID: 24555024. DOI: 10.12688/f1000research.2-9.v2

19 Czirok A and Little CD: Pattern formation during vasculogenesis. Birth Defects Res C Embryo Today 96: 153-162, 2012. PMID: 22692888. DOI: $10.1002 /$ bdrc.21010

20 Yang Y, Otte A and Hass R: Human mesenchymal stroma/stem cells exchange membrane proteins and alter functionality. Stem 
Cells Dev 24: 1205-1222, 2015. PMID: 25525832. DOI: $10.1089 / \mathrm{scd} .2014 .0413$

21 Timoshenko AV, Chakraborty C, Wagner GF and Lala PK: COX2-mediated stimulation of the lymphangiogenic factor VEGF-C in human breast cancer. Br J Cancer 94: 1154-1163, 2006. PMID: 16570043. DOI: 10.1038/sj.bjc.6603067

22 Aonuma M, Saeki Y, Akimoto T, Nakayama Y, Hattori C, Yoshitake Y, Nishikawa K, Shibuya M and Tanaka NG: Vascular endothelial growth factor overproduced by tumour cells acts predominantly as a potent angiogenic factor contributing to malignant progression. Int J Exp Pathol 80: 271-281, 1999. PMID: 10607018. DOI: 10.1046/j.1365-2613.1999.00122.x

23 Chen Z, Xu S, Xu W and Huang J: Expression of cluster of differentiation 34 and vascular endothelial growth factor in breast cancer, and their prognostic significance. Oncol Lett 10: 723-729, 2015. PMID: 2662256. DOI: 10.3892/ol.2015.3348

24 Pinto MP, Badtke MM, Dudevoir ML, Harrell JC, Jacobsen BM and Horwitz KB: Vascular endothelial growth factor secreted by activated stroma enhances angiogenesis and hormone independent growth of estrogen receptor positive breast cancer. Cancer Res 70: 2655-2664, 2010. PMID: 20332242. DOI: 10.1158/0008-5472.CAN-09-4373

25 Lin AY, Ai Z, Lee SC, Bajcsy P, Pe'er J, Leach L, Maniotis AJ and Folberg R: Comparing vasculogenic mimicry with endothelial cell-lined vessels: Techniques for 3D reconstruction and quantitative analysis of tissue components from archival paraffin blocks. Appl Immunohistochem Mol Morphol 15: 113119, 2007. PMID: 17536318. DOI: 10.1097/01 pai.0000210414. 15375.47

26 Kim HS, Won YJ, Shim JH, Kim HJ, Kim J, Hong HN and Kim BS: Morphological characteristics of vasculogenic mimicry and its correlation with EphA2 expression in gastric adenocarcinoma. Sci Rep 9: 3414, 2019. PMID: 30833656. DOI: 10.1038/s41598-019-40265-7

27 Xu S, Bai J, Zhuan Z, Li B, Zhang Z, Wu X, Luo X and Yang L: EBV-LMP1 is involved in vasculogenic mimicry formation via VEGFA/VEGFR1 signaling in nasopharyngeal carcinoma. Oncol Rep 40: 377-384, 2018. PMID: 29749553. DOI: 10.3892/or.2018.6414

28 Sun B, Zhang D, Zhao N and Zhao X: Epithelial-to-endothelial transition and cancer stem cells: two cornerstones of vasculogenic mimicry in malignant tumors. Oncotarget 8: 30502-30510, 2017. PMID: 27034014. DOI: 10.18632/oncotarget.8461

29 Leccia F, Nardone A, Corvigno S, Del Vecchio L, De Placido S, Salvatore F and Veneziani BM: Cytometric and biochemical characterization of human breast cancer cells reveals heterogeneous myoepithelial phenotypes. Cytom A 81: 960-972, 2012. PMID: 22791584. DOI: 10.1002/cyto.a.22095

30 D'Anselmi F, Masiello MG, Cucina A, Proietti S, Dinicola S, Pasqualato A, Ricci G, Dobrowolny G, Catizone A and Palombo A: Microenvironment promotes tumor cell reprogramming in human breast cancer cell lines. PLoS One 8: e83770, 2013. PMID: 24386275. DOI: 10.1371/journal.pone.0083770

31 Tsujie T, Tsujie M, Haruta Y, Barcos M, Spaulding B, Tsai H and Seon B: Differential expression of endoglin (CD105) between human malignant epithelial tissues and cultured epithelial cell lines. Cancer Res 67: 3826, 2007. PMID: 15809709. DOI: 10.3892/ijo.26.5.1193

32 Lukyanova NY, Rusetskya NV, Tregubova NA and Chekhun VF: Molecular profile and cell cycle in MCF-7 cells resistant to cisplatin and doxorubicin. Exp Oncol 31: 87-91, 2009. PMID: 19550397.

33 Zhao X, Sun B, Li Y, Liu Y, Zhang D, Wang X, Gu Q, Zhao J, Dong $X$, Liu Z and Che N: Dual effects of collagenase-3 on melanoma: metastasis promotion and disruption of vasculogenic mimicry. Oncotarget 6: 8890-8899, 2015. PMID: 25749207. DOI: 10.18632 /oncotarget. 3189

34 Uchino M, Kojima H, Wada K, Imada M, Onoda F, Satofuka H, Utsugi $\mathrm{T}$ and Murakami Y: Nuclear b-catenin and CD44 upregulation characterize invasive cell populations in nonaggressive MCF-7 breast cancer cells Masahiro. BMC Cancer 10: 414, 2010. PMID: 20696077. DOI: 10.1186/1471-2407-10-414

35 López-Knowles E, Zardawi SJ, McNeil CM, Millar EKA, Crea P, Musgrove EA, Sutherland RL and O'Toole SA: Cytoplasmic localization of $\beta$-catenin is a marker of poor outcome in breast cancer patients. Cancer Epidemiol Biomarkers Prev 19: 301309, 2010. PMID: 20056651. DOI: 10.1158/1055-9965.EPI-090741

36 Samsonraj RM, Raghunath M, Nurcombe V, Hui JH, van Wijnen AJ CS: Concise review: Multifaceted characterization of human mesenchymal stem cells for use in regenerative medicine. Stem Cells Transl Med 6: 2173-2185, 2017. PMID: 29076267. DOI: $10.1002 /$ sctm.17-0129

37 Chen WCW, Park TS, Murray IR, Zimmerlin L, Lazzari L, Huard J and Péault B: Cellular kinetics of perivascular MSC precursors. Stem Cells Int 2013: 983059, 2013. PMID: 24023546. DOI: 10.1155/2013/983059

38 Lin C-S, Xin Z-C, Dai J and Lue TF: Commonly used mesenchymal stem cell markers and tracking labels: limitations and challenges. Histol Histopathol 28: 1109-1116, 2013. PMID: 23588700. DOI: $10.14670 / \mathrm{HH}-28.1109$

39 Jaffredo T, Nottingham W, Liddiard K, Bollerot K, Pougeta C and de Bruijnb M: From hemangioblast to hematopoietic stem cell: An endothelial connection? Exp Hematol 33: 1029-1040, 2005. PMID: 16140151. DOI: 10.1016/j.exphem.2005.06.005

40 Trkov S, Eng G, Di Liddo R, Parnigotto PP and VunjakNovakovic G: Micropatterned 3-dimensional hydrogel system to study human endothelial-mesenchymal stem cell interactions. J Tissue Eng Regen Med 4: 205-215, 2010. PMID: 19998330. DOI: $10.1002 /$ term. 231

41 Slukvin II and Kumar A: The mesenchymoangioblast, mesodermal precursor for mesenchymal and endothelial cells. Cell Mol Life Sci 75: 3507-3520, 2018. PMID: 29992471. DOI: 10.1007/s00018-018-2871-3

42 Otranto M, Sarrazy V, Bonté F, Hinz B, Gabbiani G and Desmoulière A: The role of the myofibroblast in tumor stroma remodeling. Cell Adh Migr 6: 203-219, 2012. PMID: 22568985. DOI: $10.4161 / \mathrm{cam} .20377$

43 Mark P, Kleinsorge M, Gaebel R, Lux CA, Toelk A, Pittermann E, David R, Steinhoff G and Ma N: Human mesenchymal stem cells display reduced expression of CD105 after culture in serum-free medium. Stem Cells Int 2013: 698076, 2013. PMID: 24194767. DOI: $10.1155 / 2013 / 698076$

44 Pham LH, Vu NB and Pham P Van: The subpopulation of CD105 negative mesenchymal stem cells show strong immunomodulation capacity compared to CD105 positive mesenchymal stem cells. Biomed Res Ther 6: 3131-3140, 2019. DOI: $10.15419 /$ bmrat.v6i4.538

45 Saidova AA and Vorobjev IA: Lineage commitment, signaling pathways, and the cytoskeleton systems in mesenchymal stem 
cells. Tissue Eng Part B Rev 26: 13-25, 2020. PMID: 31663422. DOI: $10.1089 /$ ten .teb.2019.0250

46 Dellagi K, Vainchenkerl W, Vinci G, Paulin D and Brouet JC: Alteration of vimentin intermediate filament expression during differentiation of human hemopoietic cells. EMBO J 2: 15091514, 1983. PMID: 11892803.

47 Rezaeian L, Hosseini SE, Dianatpour M, Edalatmanesh AM, Tanideh N, Mogheiseh A and Tamadon A: Intrauterine xenotransplantation of human Wharton jelly-derived mesenchymal stem cells into the liver of rabbit fetuses: A preliminary study for in vivo expression of the human liver genes. Iran J Basic Med Sci 21: 89-96, 2018. PMID: 29372042. DOI: $10.22038 / \mathrm{ijbms} .2017 .24501 .6098$
48 Tomiyama L, Kamino H, Fukamachi H and Urano T: Precise epitope determination of the anti-vimentin monoclonal antibody V9. Mol Med Rep 16: 3917-3921, 2017. PMID: 28765898. DOI: $10.3892 / \mathrm{mmr} .2017 .7102$

49 Yu S-J, Kim H-J, Lee ES, Park C-G, Cho SJ and Jeon S-H: $\beta$ catenin accumulation is associated with increased expression of nanog protein and predicts maintenance of MSC self-renewal. Cell Transplant 26: 365-377, 2017. PMID: 27684957. DOI: $10.3727 / 096368916 \times 693040$

Received June 22, 2020

Revised July 10, 2020

Accepted July 16, 2020 